\title{
Photocrosslinking of Thioxanthone Group Containing Copolymers for Surface Modification and Bioanalytics
}

Simon Zunker, Jürgen Rühe*

IMTEK - Department of Microsystems Engineering, Laboratory for Chemistry and Physics of Interfaces, University of Freiburg, Georges-Koehler-Allee 103, 79110 Freiburg, Germany

Center of Excellence @ Freiburg Center for Interactive Materials and Bioinspired Technologies; University of Freiburg, Georges-Koehler-Allee 105, 79110 Freiburg, Germany

\section{Supplemental Information}

${ }^{1} \mathrm{H}-\mathrm{NMR}$ spectra of the thioxanthone compounds and polymers synthesized in this study.

1. 2-Hydroxythioxanthone

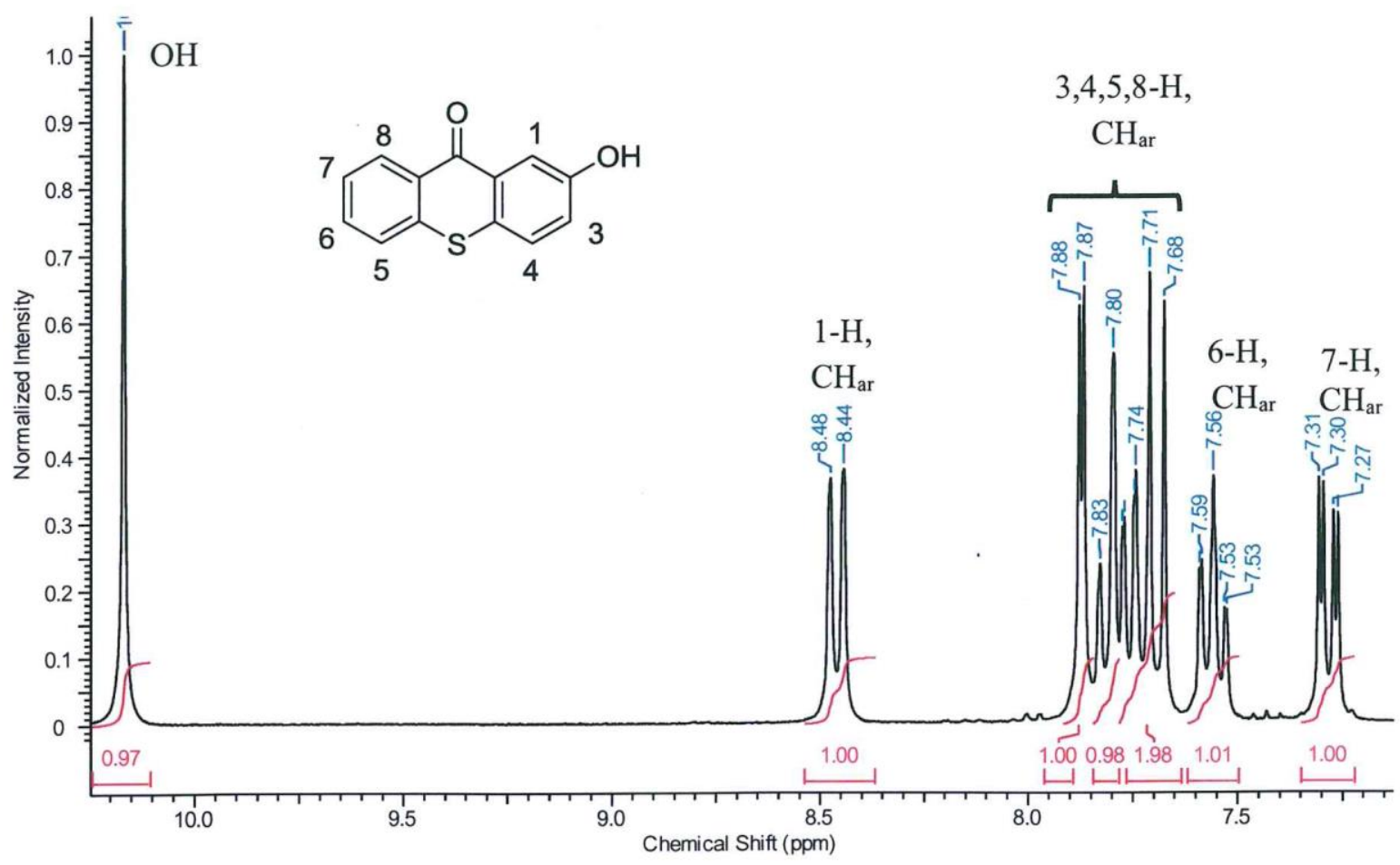


2. 2-Acryloyloxythioxanthone

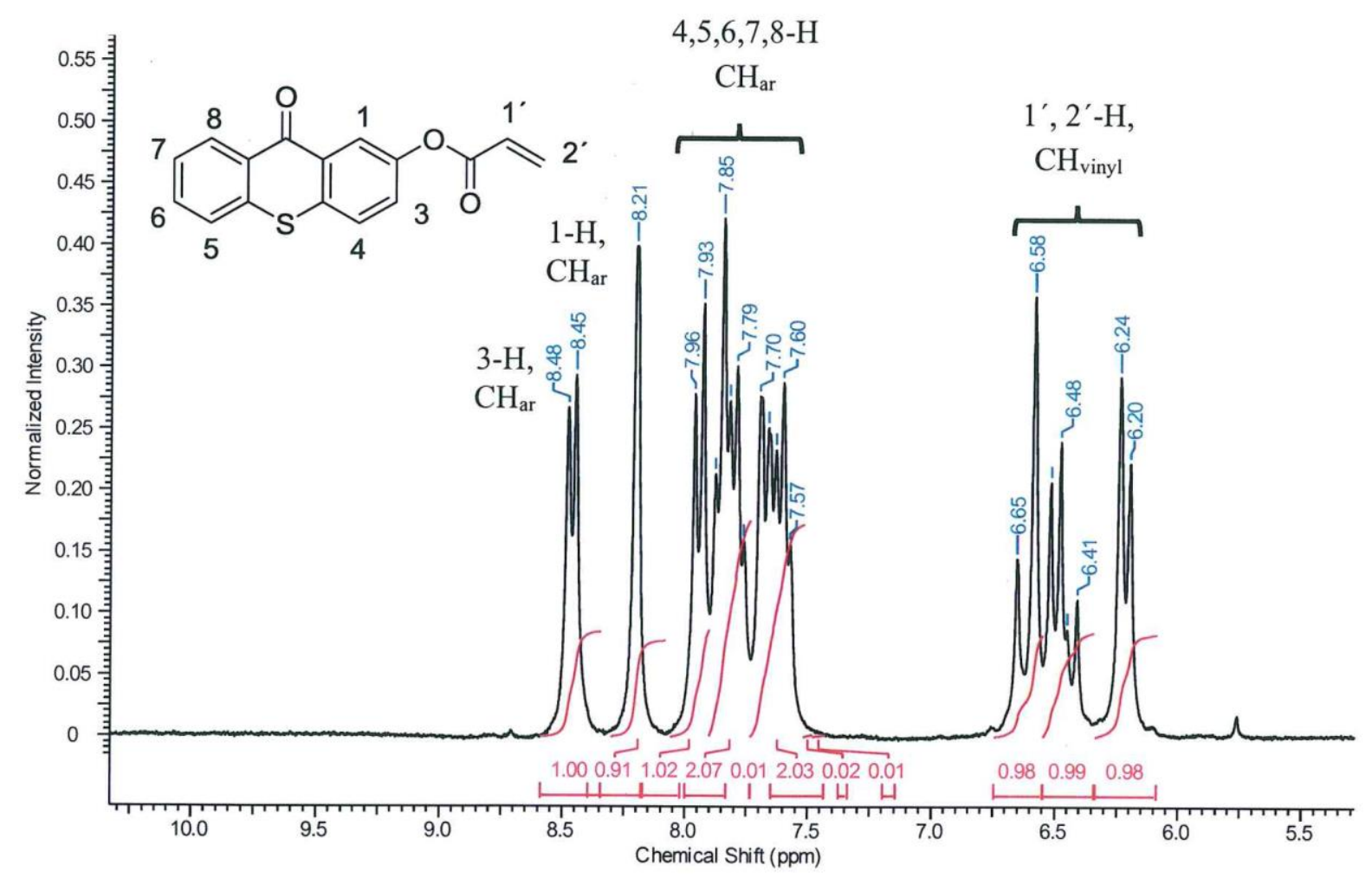

3. PMMA-co-AOTX-co-DMAMA (4.6\% AOTX, 11\%DMAMA)

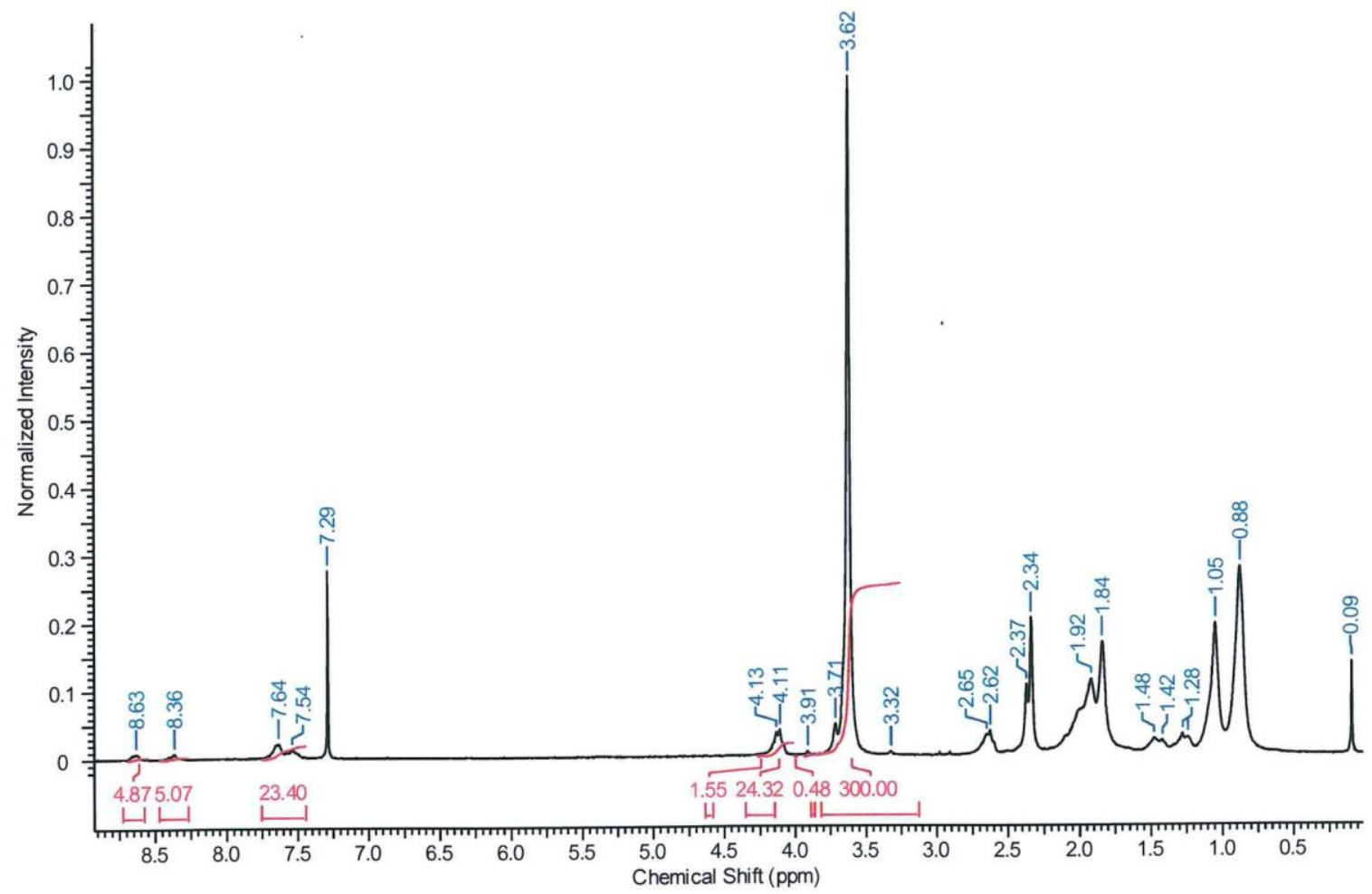

